\title{
Request Satisfaction Problem in Synchronous Radio Networks
}

\author{
Benoît Darties, Sylvain Durand, and Jérôme Palaysi \\ LIRMM, Université Montpellier II \\ 161 rue Ada, 34392 Montpellier Cedex 5 - France \\ \{benoit.darties, sylvain.durand, jerome.palaysi\}@lirmm.fr
}

\begin{abstract}
We study two algorithmical problems inspired from routing constraints in a multihop synchronous radio network. Our objective is to satisfy a given set of communication requests in the following model: nodes send omnidirectional radio transmissions in synchronous slots; during a given slot, a node can receive a message from an adjacent node if and only if no other neighbour is transmitting - otherwise, radio interferences may occur if two or more neighbors transmit in the same slot -. The objective is to minimize the number of slots used. The two problems differ in that the routing policy may be imposed (DAWN-path), or not (DAWN-request). In this second case, a path must be assigned for each request, to define the nodes to use to reach the destination from the source. We present some complexity results, in particular showing that both problems are NP-hard when the network is restricted to a tree. We also present a polynomial algorithm in $O\left(n^{2 K}\right)$ when the number of requests is bounded (by above) by a constant $K$.
\end{abstract}

Key words: Radio Network, Request Satisfaction, Complexity

\section{Introduction}

A radio network is a collection of transmitter-receiver stations (or nodes) communicating with one another via multihop wireless links. The use of the radio medium implies some restrictions and properties: whenever a node transmits, all the nodes in its communication range may receive the transmission. Incoming messages have to be forwarded to reach nodes which are located more than one hop away from the source. Since all nodes share the same frequency channel, a collision may occur if two or more neighbors transmit simultaneously, preventing correct reception of the message.

In this paper, we study two communication problems inspired from routing constraints in this kind of network. We consider the following simplified communication model, which has been widely used for the broadcast problem[1,4-6, $10,12,13]$ or the gathering problem $[2,3]$ in multihop radio networks : nodes send messages in synchronous slots; during each slot each node acts either as a transmitter or a receiver. A node acting as a transmitter sends a message which can potentially reach the nodes that are in its communication range. A node 
acting as a receiver successfully receives a message from a transmitter node if no other neighbor transmits in this slot. If two or more neighbors of a receiver node $u$ transmit simultaneously in a given slot, then the messages may interfere with each others (collide) and the messages are not transmitted successfully to $u$. We note that two neighbors $u$ and $v$ may successfully transmit in the same slot to $u^{\prime}$ and $v^{\prime}$, if we assume that $u^{\prime}$ and $v$ are not adjacent, and respectively $v$ and $u^{\prime}$ : the node $u$ acting as transmitter simply ignores the transmission of $v$ and reciprocally. Such a network is characterized as a $\Delta$-port transmission, 1port reception, half-duplex, synchronous network. We suppose that the network topology is fixed, at least during the time the problem must be solved. All those properties specify the model we use in this work.

In this context, we consider the problem of satisfying a set of communication requests within a minimum timeframe, by indicating for each node the slots on which it has to relay transiting packets. A request is a couple of sourcedestination nodes representing the starting and ending nodes of a given message. The second section of this paper details the model and introduces the DAWNpath and DAWN-request problems. General complexity results are discussed in a third section where we will show that these problems are quite difficult even for particular cases. However we present in the fourth section a polynomial algorithm when the number of requests is bounded by a constant $K$.

\section{Describing the model and expressing the problem}

\section{$2.1 \quad$ The model}

The network is represented as an undirected graph $G$ where the set $V(G)$ of vertices corresponds to the set of nodes of the network. An edge $e=\{u, v\} \in E(G)$ denotes that $u$ can directly communicate to $v$ (no additional node is required to relay the message) and reciprocally ${ }^{1}$.

A request $r$ is a couple $(s, t) \mid s, t \in V(G)$, where $s$ represents the source and $t$ the destination of the request.

A path of length $k$ in a graph $G$ is an ordered list $\left(v_{0}, v_{1}, \ldots, v_{k}\right)$, where $v_{i} \in V(G)$ for any $i \in[0, k]$, and such that the edge $\left(v_{i}, v_{i+1}\right)$ exists in $E(G)$ for any $i \in[0, k-1]$, and all the edges are different. Throughout the paper all the considered paths are simple paths, that is, paths which visit a vertex at most once. Paths are used here to represent a communication road in the network.

Given a graph $G$ and a collection of communication requests $R$, let $P$ be a routing function on $R$ which associates to each $r=(s, t) \in R$ a path $P(r)$ in $G$, (also denoted by $P_{r}$ ), beginning with $s$ and ending with $t$.

Given a graph $G$, a collection of requests $R$, and a routing function $P$, we define a date assignment $d$ to be a function which takes two arguments $r$ and $x$, with $r=(s, t) \in R, x \in P_{r}$ and $x \neq t$, and returns an integer $d(r, x)$. This

\footnotetext{
${ }^{1}$ We consider that if $x$ can directly communicate with $y$ then $y$ can directly communicate with $x$. We can deduce that the graph is symmetrically directed, and will be represented by an undirected graph.
} 
integer corresponds to a slot, such that $x$ transmits the message of $r$ to the next hop during this slot. Multiplexing is not allowed, this implies that each transmission only contains a single message. A date assignment $d$ is said to be valid if and only if for each request $r=\left(x_{0}, x_{k}\right)$ with $P_{r}=\left(x_{0}, x_{1}, \ldots, x_{k}\right)$ the proposition $d\left(r, x_{0}\right)<d\left(r, x_{1}\right)<\ldots<d\left(r, x_{k-1}\right)$ is true. Moreover we say that a valid assignment is conflict-free if and only if for each $d\left(r, x_{i}\right)=d\left(r^{\prime}, y_{j}\right)$ where $r \neq r^{\prime}$, the following holds :

1. $x_{i} \neq y_{j}$ : prevents multiplexing

2. $x_{i+1} \neq y_{j} \wedge y_{j+1} \neq x_{i}$ : a node cannot receive and transmit simultaneously

3. $\left\{x_{i}, y_{j+1}\right\} \notin E(G) \wedge\left\{y_{j}, x_{i+1}\right\} \notin E(G): \Delta$-port-transmission, 1-port-reception

We note $\max (d)=\max _{r \in R, x \in V(G)} d(r, x)$ the cost of $d$, i.e. the number of slots used by a date assignment $d$.

\subsection{The DAWN problem}

Given a set of requests to satisfy in a synchronous radio network and a maximum number of slots, the problem DAWN (Date Assignment in Wireless Network) consists in finding a conflict-free date assignment along communication paths. According to whether the paths are given (e.g. by the routing function) or not, we distinct two main problems: DAWN-paths and DAWN-request.

The $D A W N$-path problem is stated as follows:

INPUT: An undirected graph $G$, a collection of requests $R=\left\{r_{i}=\left(s_{i}, t_{i}\right) \mid 1 \leq\right.$ $i \leq K\}$, a routing function $P$ on $R$ which associates to each request $r_{i}$ a path $P\left(r_{i}\right)$ linking the vertices of $r_{i}$, a natural integer $D$.

QUESTION: Does a valid and conflict-free date assignment exist in such a manner that the number of required slots is lower than or equal to $D$ ?

Let min-DAWN-path be the optimization version of DAWN-path. For each natural integer $D$ we define the $D$-DAWN-path problem as the subclass of DAWN-path where the maximum number of allowed slots is $D$. Note that $D$ is bounded by above by $|V(G)| \times|R|$ otherwise the answer is obviously "yes". Figure 1 presents an instance of DAWN-path (1(a)) and a solution (conflict-free assignment) to it (1(b)).

We observe that there is no optimal fixed routing for the DAWN-path problem [8, page 97]. This leads us to propose the DAWN-request problem:

INPUT: An undirected graph $G$, a collection of requests $R=\left\{r_{i}=\left(s_{i}, t_{i}\right) \mid 1 \leq\right.$ $i \leq K\}$, a natural integer $D$.

QUESTION: Does a valid and conflict-free date assignment exist in such a manner that the number of required slots is lower than or equal to $D$ ?

As stated before, we call min-DAWN-request the optimization version of DAWN-request, and $D$-DAWN-request the subclass of DAWN-request where $D$ is the maximum number of allowed slots. 


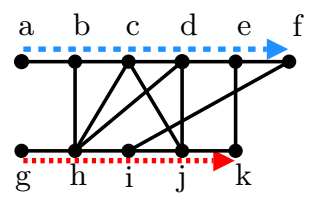

(a)

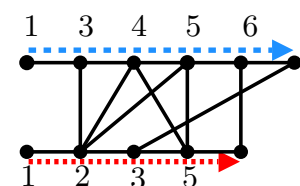

(b)

Fig. 1. An instance $(G, R, P)$ of min-DAWN-path containing 2 requests $r_{1}=(a, f)$, $r_{2}=(g, k), P\left(r_{1}\right)=(a, b, c, d, e, f)$ and $P\left(r_{2}\right)=(g, h, i, j, k)$ (sub-fig. a). A valid and conflict-free date assignment and within a minimum number of slots (sub-fig. b).

\section{Complexity results}

We adopt the terminology of [7]: a problem is not approximable within a constant factor if no polynomial approximation algorithm with a constant performance guarantee exists. Moreover an approximation algorithm has a constant performance guarantee of $\rho$ if for each instance $I$ of a problem it finds a solution the cost of which is at most $\rho$ times the cost of the optimal solution for instance $I$.

In the following subsection we show that in general min-DAWN-path and min-DAWN-request are NP-hard and not approximable within a constant factor. These results are based on the complexity of coloring problems on graphs. The DCOLORING problem [11] consists in assigning a color (represented by a number bounded by above by $D$ ) to each vertex assuming that two adjacent vertices are assigned different colors. It is known that D-COLORING is NP-complete for any constant $D \geq 3$, and that the corresponding minimization problem minCOLORING is NP-hard and not approximable within a constant factor.

In a second subsection we show these problems remain NP-hard even when the network is a tree, but here the reduction does not enable us to prove the inapproximability (within some constant). In the third subsection we locate the boundaries between polynomiality and NP-completeness for $D$-DAWN-path and $D$-DAWN-request when only $D$ varies.

\subsection{Two difficult problems}

The first theorem proves the NP-completeness of both problems in general.

Theorem 1. Problems min-DAWN-path and min-DAWN-requests are NP-hard and not approximable within some constant factor. For any $D \geq 3$, decision problems D-DAWN-path and D-DAWN-request are NP-complete.

Proof. We first prove the NP-completeness of D-DAWN-request by a reduction to D-COLORING.

D-DAWN-request is in NP : given a routing function $P$ and a date assignment $d$ as a solution of an instance, one can check in a polynomial time if $P$ enables 
each message to reach their destination, and if $d$ is a valid conflict-free date assignment using fewer than $D$ slots.

Let $I_{C}=\left(G_{C}\right)$ be an instance of D-COLORING, and let us note $n=$ $\left|V\left(G_{C}\right)\right|$. From $I_{C}$ we define an instance $I=(G, R)$ of D-DAWN-request, where $G$ is a graph such that $V(G)=\left\{s_{x}, t_{x} \mid x \in V\left(G_{C}\right)\right\}$ and $E(G)=\left\{\left\{s_{x}, t_{x}\right\} \mid x \in\right.$ $\left.V\left(G_{C}\right)\right\} \cup\left\{\left\{s_{x}, t_{y}\right\} \mid\{x, y\} \in E\left(G_{C}\right)\right\} \cup\left\{\left\{t_{x}, t_{y}\right\} \mid x, y \in V\left(G_{C}\right)\right\}$. We define the set $R=\left(r_{x}=\left(s_{x}, t_{x}\right) \mid x \in V\left(G_{C}\right)\right)$ of $n$ communication requests. Clearly, the instance $I$ can be constructed in a polynomial time. Figure 2(b) gives an example of a graph $G$ constructed from the graph $G_{C}$ of figure $2($ a).

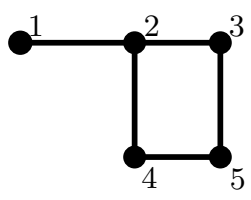

(a) A graph $G_{C}$

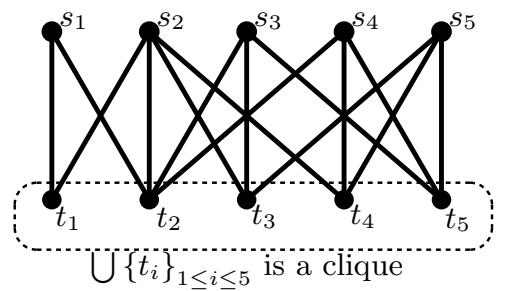

(b) The resulting graph $G$

Fig. 2. Construction of $G$ from $G_{c}$

We show that, if there exists a valid conflict-free date assignment for $I$ using $k<D$ slots, then there exists a solution to the instance $I_{C}$ of D-COLORING with cost $k \leq D$ and reciprocally.

Let $S=(P, d)$ be a solution of $I=(G, R)$ where $P$ is a routing function for $R$ and $d$ a valid and conflict-free date assignment for $(G, R, P)$ with cost $k=\max (d) \leq D$. Let us suppose there exists a request $r_{i}=\left(s_{i}, t_{i}\right)$ in $S$ such that the message is not directly emitted from $s_{i}$ to $t_{i}$, but requires at least one relay node $t_{j} \mid j \neq i$. If $t_{j}$ transmits in slot $u$, then no other node $s_{l}$ may transmit in the same slot, while $\cup_{i<n} t_{i}$ is a clique. Then we can extract a solution $S^{\prime}$ from $S$ with cost $z^{\prime} \leq z$ in which $s_{i}$ transmits directly to $t_{i}$ at slot $u$. Thus from any solution $S$ with cost $k$, we compute a proper solution $S^{\prime}=\left(P^{\prime}, d^{\prime}\right)$ with cost $k^{*} \leq k \leq D$ such that each message is directly transmitted from its source to its destination. Clearly $P^{\prime}\left(r_{i}\right)=\left(s_{i}, t_{i}\right) \forall r_{i} \in R$. Let $c$ be the function which assigns to each vertex $x \in V\left(G_{c}\right)$ the color $d^{\prime}\left(r_{x}, s_{x}\right)$. Let us note that $\max \left(d^{\prime}\right)=\max (c) \leq D$. The resulting coloring is valid because if $x$ and $y$ are adjacent in $G_{c}$ then by construction the edges $\left\{r_{x}, t_{y}\right\}$ and $\left\{r_{y}, t_{x}\right\}$ exist in $G$ and imply that $d\left(r_{x}, s_{x}\right) \neq d\left(r_{y}, s_{y}\right)$.

Reciprocally, let $c$ be a vertex coloring of $G_{c}$ with cost $k \leq D$. Let $P$ the routing function such that $P\left(r_{i}\right)=\left(s_{i}, t_{i}\right) \forall r_{i} \in R$, and $d$ be the date assignment which associates the date $c(x)$ to each couple $\left(r_{x}, s_{x}\right)$. Then $d$ is a valid conflictfree date assignment such that $\max (c)=\max (d)$. 
To conclude, we claim that to any vertex coloring $c$ of $I_{C}$ corresponds a solution to $I$ composed of a routing function $P$ a valid and conflict-free date assignment $d$ of $(G, R)$ such that $\max (c)=\max (d)$, and reciprocally. Since DCOLORING is NP-complete for any $D \geq 3[11]$ and D-DAWN-request belongs to NP, then D-DAWN-request is also NP-complete for any $D \geq 3$. This proof can be extended to prove the NP-completeness of D-DAWN-path for any $D \geq 3$, by adding to the instance $I$ the routing function $P$ such that $P\left(r_{i}\right)=\left(s_{i}, t_{i}\right) \forall r_{i} \in R$. By adapting this proof to the optimization versions of these problems we show that min-DAWN-path and min-DAWN-request are NP-hard by a reduction to min-COLORING. Therefore the reduction preserves the inapproximability of min-COLORING, which is NP-hard and not approximable within some constant factor. This allows to conclude.

We now show that DAWN-path and DAWN-request are still NP-complete even if the network is a tree. This resolves an open question suggested in [9]. The proof can be extended to binary tree or Unit Disk Graph (intersection graph of disks with equal diameters). UDG are often used to model the topology of ad-hoc wireless communication networks. Let us introduce the following propositions:

Lemma 1. Let $I=(G, R, P, D)$ be an instance of $D A W N$-path, let $x$ a vertex from $V(G)$ and $i$ an integer. Then there exists an instance $I^{\prime}=\left(G^{\prime}, R^{\prime}, P^{\prime}, D\right)$ of the same problem with $V(G) \subseteq V\left(G^{\prime}\right), R \subseteq R^{\prime}$, such that :

- each valid and conflict-free date assignment d on $I^{\prime}$ requires exactly $D$ slots, and is also a valid and conflict-free date assignment on $I$,

- for each valid and conflict-free date assignment $d$ on $I^{\prime}$ and each request $r \in R^{\prime}$ we have $d(r, x) \neq i$,

- the instance I can be constructed in a polynomial time.

Proof. Let us consider the following items:

- an instance $I=(G, R, P, D)$ of DAWN-path,

- a request $r \in R$ such that $P(r)=(s, \ldots, x, y, \ldots, t)$,

- a chain $C=\left\{c_{1}, c_{2}, c_{3}, \ldots, c_{D+1}\right\}$ of length $D+1$, with $V(G) \cap V(C)=\emptyset$,

- a request $r^{\prime}=\left(c_{1}, c_{D+1}\right)$,

- a natural integer $i \in[1, D]$.

We note $H=\left(V(G) \cup V(C), E(G) \cup E(C) \cup\left\{\left\{y, c_{i}\right\}\right\}\right)$ and define $P\left(r^{\prime}\right)=$ $\left(c_{1}, c_{2}, c_{3}, \ldots, c_{D+1}\right)$. Note that if $G$ is a tree, then $H$ is also a tree. Figure 3 schematically illustrates such a construction. We claim the following :

1. The instance $I^{\prime}=\left(H, R \cup\left\{r^{\prime}\right\}, P, D\right)$ can be constructed in polynomial time from $I=(G, R, P, D)$,

2. let $d$ be a valid and conflict-free date assignment for $I^{\prime}$, then we have $d\left(r^{\prime}, c_{i}\right)=i, d(r, x) \neq i$, and $d$ is a valid date assignment for $I$.

This construction presented in the proof of lemma 1 will be used in the proof of theorem 2 to prevent some nodes from transmitting during certain slots. In 


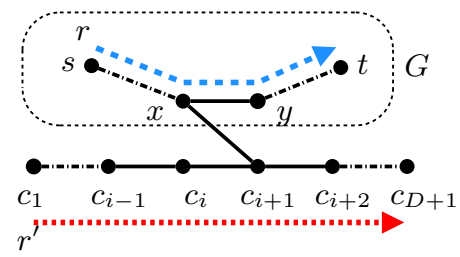

Fig. 3. How to prevent a node $x$ from transmitting in slot $i$

the following, we say that a request $r=(s, t)$ starts at slot $t$ if the source node $s$ proceeds to the transmission of the message of $r$ during the $t$ th time slot.

Let $u$ and $D$ be two natural integers such that $D \geq 6$. We define a tight $(u, D)$ DAWN-path instance as the DAWN-path instance $\left(C_{[1, D+7 u]}, R, P, D\right)$ where $C_{[1, D+7 u]}$ is a chain having $D+(7 u)$ vertices $\{1,2, \ldots, D+7 u\}$ and the edges $\{i, i+1\} \mid \forall 0 \leq i \leq D+7 u-1 . R$ is the set of $2 u$ requests $\left\{r_{1}, \bar{r}_{1}, r_{2}, \bar{r}_{2}, \ldots, r_{u}, \bar{r}_{u}\right\}$ with $r_{i}=\{(7 i-5,7 i+D-9)\}$ and $\bar{r}_{i}=\{(7 i-6,7 i+D-8)\} \mid \forall i \leq u$.

Lemma 2. Let us consider a tight $(u, D)$ instance for some integer $u$ and $D$. Let us suppose $d$ is a valid and conflict-free date assignment, and $i \in[1, u]$. We make the following observations:

1. $\left(d\left(r_{i}, 7 i-5\right), d\left(\bar{r}_{i}, 7 i-6\right)\right) \in\{(5,1),(1,3)\}$,

2. $d\left(r_{i}, j+1\right)=d\left(r_{i}, j\right)+1, \forall j \in P\left(r_{i}\right)-\{7 i+D-9\}$,

3. $d\left(\bar{r}_{i}, j+1\right)=d\left(\bar{r}_{i}, j\right)+1, \forall j \in P\left(\bar{r}_{i}\right)-\{7 i+D-8\}$.

Given two natural integers $i \in[1, u]$ and $j \in P\left(r_{i}\right)$ :

1. if $d\left(r_{i}, 7 i-5\right)=1$ then $d\left(r_{i}, j\right)=j-7 i+6$ and $d\left(\bar{r}_{i}, j\right)=j-7 i+9$

2. if $d\left(r_{i}, 7 i-5\right)=5$ then $d\left(r_{i}, j\right)=j-7 i+10$ and $d\left(\bar{r}_{i}, j\right)=j-7 i+7$

Proof. This obvious proof is left to the reader.

Theorem 2. DAWN-path and DAWN-request remain NP-complete even if the graph representing the network topology is a tree.

Proof. The proof is based on a polynomial reduction of any instance of 3-SAT problem [11] to an instance $(G, R, P, D)$ of DAWN-path where $G$ is a tree.

Let $I_{S A T}=(U, W)$ be an instance of 3-SAT, composed of a set of variables $U=\left\{x_{1}, x_{2}, \ldots, x_{n}\right\}$ and a set of clauses of 3 literals $W=\left\{c_{1}, c_{2}, \ldots, c_{m}\right\}$. We note $n=|U|, m=|W|$, and set $D=m+7 n+3$.

Let us consider the tight $(n, D)$ instance $\left(G_{1}, R_{1}, P, D\right)$. The main idea of the proof consists in assigning two requests $r_{i}$ and $\bar{r}_{i}$ to each variable $x_{i} \in W$. Thus for the sake of clarity we note $r_{x_{i}}$ the request $r_{i}$ and $r_{\bar{x}_{i}}$ the request $\bar{r}_{i}$.

Let $G_{2}$ be the tree such that $V\left(G_{2}\right)=V\left(G_{1}\right) \cup\left(c_{i}, 1\right),\left(c_{i}, 2\right) \mid i \in[1, m]$ and $E\left(G_{2}\right)=E\left(G_{1}\right) \cup\left\{\left\{\left(c_{i}, 1\right),\left(c_{i}, 2\right)\right\},\left\{7 n+i, c_{i}^{s}\right\} \mid i \in[1, m]\right\}$. In the following we note $c_{i}^{s}$ the couple $\left(c_{i}, 1\right)$ and $c_{i}^{t}$ the couple $\left(c_{i}, 2\right)$ for any integer $i$. Let $R_{2}$ 


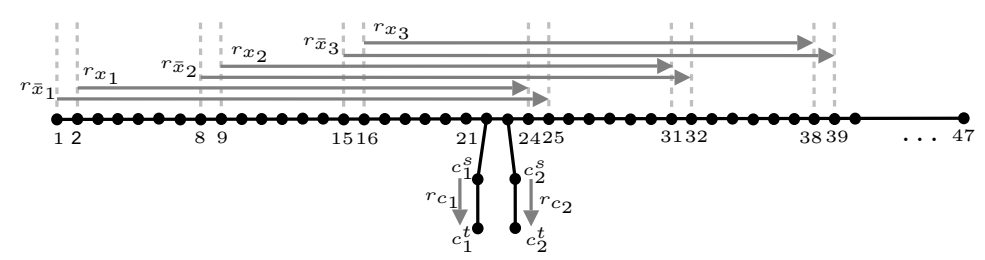

Fig. 4. A graph $G_{2}$ and a set of requests $R_{1} \cup R_{2}$ constructed from an instance $I_{S A T}=$ $(U, W)$ where $U=\left\{x_{1}, x_{2}, x_{3}\right\}$ and $W=\left\{c_{1}, c_{2}\right\}$. Here $D=26$.

be the set of requests $\left\{r_{c_{i}}=\left(c_{i}^{s}, c_{i}^{t}\right) \mid i \in[1, m]\right\}$. Now, consider the instance $\left(G_{2}, R_{1} \cup R_{2}, P, D\right)$ (see Figure 4 for an example).

By applying the construction of lemma 3.1 several times, we create an instance $I=(G, R, P, D)$ by adding elements to $\left(G_{2}, R_{1} \cup R_{2}, P, D\right)$ as follows : for each request $r_{c_{i}}=\left(c_{i}^{s}, c_{i}^{t}\right)$ we prevent $c_{i}^{s}$ from transmitting to $c_{i}^{t}$ the message of request $r_{c_{i}}$ during each slot $t \leq D$, except for 3 specific ones which are defined from the literals of the clause $c_{i}$ : if $c_{i}$ contains the positive (resp. negative) literal associated to the variable $x_{j} \mid j \leq n$, then $c_{i}^{s}$ is allowed to transmit in slot $7 n+i-7 j+6$ (resp. $7 n+i-7 j+8)$. Since $G_{2}$ is a tree, $G$ is also a tree and the routing function is still obvious.

The size of $I$ is polynomial in the size of $I_{S A T}$, and it can be constructed in a polynomial time. We claim that if there is a solution in $D$ slots to $I$, then we can deduce a solution to the instance $I_{S A T}$ and reciprocally.

Let us consider a valid and conflict-free date assignment $d$ on the instance $I$. According to lemma 2 and for each integer $i \in[1, n]$, one request of $\left\{r_{x_{i}}, r_{\bar{x}_{i}}\right\}$ must start at slot 1 , and the other as soon as possible, and once a request has been started, its progression cannot be stopped. Moreover each request $r_{c_{i}} \mid i \in[1, m]$ is clearly satisfied by $d$. The slot which has been assigned to $\left(r_{c_{i}}, c_{i}^{s}\right)$ is one of the three allowable values defined from the literals of clause $c_{i}$. Our construction implies that there exists $j$ such that $d\left(r_{c_{i}}, c_{i}^{s}\right)$ is of the form $7 n+i-7 j+6$ or $7 n+i-7 j+8$, according to $x_{j}$ is a positive or a negative literal. If $x_{j}$ is a positive literal, then the source of $r_{x_{j}}$ is located on vertex $7 j-5$, i.e. at distance $7 n+i_{7} j+5$ from the vertex $7 n+j$ which is adjacent to $c_{i}^{s}$. Then $r_{x_{j}}$ necessarily starts at slot 1 - and we have $d\left(r_{x_{j}}, 7 n+i\right)=d\left(r_{c_{i}}, c_{i}^{s}\right)$ - otherwise messages would collide. We adopt a similar reasoning when $x_{j}$ is a negative literal. Then for each clause $c_{i}$, there exists at least one (positive or negative) literal $l \in c_{i}$, such that the request $r_{l}$ starts before $r_{\bar{l}}$. By affecting the value "True" to all variables $x_{i}$ where $r_{x_{i}}$ has been started at slot 1 (i.e. before $r_{\bar{x}_{i}}$ ) and "False" otherwise, we obtain a solution to the instance $I_{S A T}$.

Reciprocally we can deduce a solution to the instance $I$ from a solution to $I_{S A T}$ : for each variable $x_{i}$ we start the request $r_{x_{i}}$ before $r_{\bar{x}_{i}}$ if and only if $x_{i}$ has the value "True". For each clause $c_{i}$, we start $r_{c_{i}}$ at the first valid and available slot (this slot exists since the clause $c_{i}$ is satisfied by at least one literal). 
To conclude we point out that 3-SAT is NP-complete and that DAWN-path belongs to NP. Then the DAWN-path is NP-complete even if the network is a tree. This implies the NP-completeness of DAWN-request, since there is one unique path linking each source to its destination when the network in a tree.

\subsection{Locating the boundaries between polynomiality and NP-completeness}

We have shown that DAWN-path and DAWN-request are NP-complete even when the network topology is very restrictive. In the following subsection, we focus our interest on the influence of the maximum number of slots $D$ on the complexity of these problems.

Theorem 1 already affirms that D-DAWN-path and D-DAWN-request are NP-complete when $D \geq 3$. By the way when $D=1$ one can verify in polynomial time if an instance can be satisfied: for each request $r=(s, t), s$ must be adjacent to $t$, and can only emits at the first slot. Hence $t$ cannot be adjacent to another source node $s_{2}$, and $s$ must be a source only for $t$. The next theorem states that one can check in a polynomial time if there is a solution to a D-DAWN-path instance when $D \leq 2$.

Theorem 3. The 2-DAWN-path decision problem is polynomial

Proof. Let $I=(G, R, P)$ an instance of 2-DAWN-path. One can suppose that for each request $r=(s, t)$ the path $P(r)$ contains at most one vertex $l$ between $s$ and $t$, otherwise the instance is clearly insolvable. We propose an algorithm in three steps :

- Dates are forced for transmitters belonging to two-hop requests.

- Therefore dates 1 (resp. 2) are spread to every transmitter which cannot transmit during the second (resp. first) slot.

- Remaining dates are computed using a 2-SAT-like algorithm.

Theorems 1 and 3 show that D-DAWN-path is polynomial when the maximum number of slots $D$ is lower than or equal to 2 , and becomes NP-complete when $D \geq 3$. Theorem 4 proves that D-DAWN-request is NP-complete for $D=2$.

Theorem 4. The 2-DAWN-request decision problem is NP-complete

Proof. Let $I_{S A T}=(U, W)$ be an instance of 3-SAT where $U=\left\{x_{1}, \ldots, x_{n}\right\}$ denotes a set of variables and $W=\left\{c_{1}, \ldots, c_{m}\right\}$ a set of clauses. For each variable $x_{i} \in U$ let $H_{x_{i}}=(X, Y, E)$ be the complete bipartite graph $K_{3,2}$ such that $X=\left\{\left(1, x_{i}\right),\left(1, \bar{x}_{i}\right)\right\}$ and $Y=\left\{\left(2, x_{i}\right),\left(2, \bar{x}_{i}\right\}\right.$. For each clause $c_{i}=$ $\left\{l_{1}, l_{2}, l_{3}\right\} \in W$, let $F_{c_{i}}=(X, Y, E)$ be the complete bipartite graph $K_{3,2}$ such that $X=\left\{\left(1, c_{i}\right),\left(2, c_{i}\right)\right\}$ and $Y=\left\{\left(c_{i}, l_{1}\right),\left(c_{i}, l_{2}\right),\left(c_{i}, l_{3}\right)\right\}$. Note that $l_{1}$ to $l_{3}$ can be positive or negative literals, each literal corresponding to a variable $x_{i} \in U$. Let $I=(G, R)$ be a 2-DAWN-request instance with $V(G)=\left\{\left\{\bigcup_{x_{i} \in U} V\left(H_{x_{i}}\right)\right\} \cup\right.$ $\left.\left\{\bigcup_{c_{i} \in W} V\left(F_{c_{i}}\right)\right\}\right\}$ and $E(G)=\left\{\left\{\bigcup_{x_{i} \in U} E\left(H_{x_{i}}\right)\right\} \cup\left\{\bigcup_{c_{i} \in W} E\left(F_{c_{i}}\right)\right\} \cup\left\{\left(c_{i}, l\right),(2, l)\right.\right.$ 
$\left.\left.\mid c_{i} \in W, l \in c_{i}\right\}\right\}$. Figure 5 presents an example of graph $G$ constructed from a 3-SAT instance $I_{S A T}=(U, W)$. The requests collection $R$ contains exactly all the requests of the form $\left(\left(1, c_{i}\right),\left(2, c_{i}\right)\right) \mid c_{i} \in W$, and $((1, l),(2, l))$ where $l$ is a literal corresponding to a variable $x_{i} \in U$.

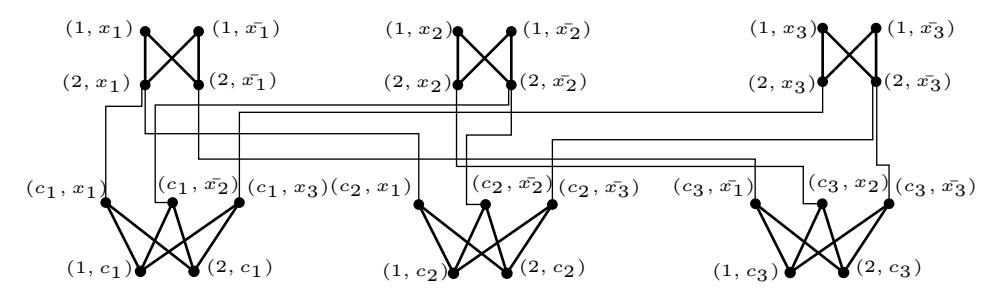

Fig. 5. graph constructed from $I_{S A T}=(U, W)$ with $U=\left\{x_{1}, x_{2}, x_{3}\right\}$ and $W=$ $\left\{c_{1}, c_{2}, c_{3}\right\}$ with $c_{1}=\left\{x_{1}, \overline{x_{2}}, x_{3}\right\}, c_{2}=\left\{x_{1}, \overline{x_{2}}, \overline{x_{3}}\right\}$ and $c_{3}=\left\{\overline{x_{1}}, x_{2}, \overline{x_{3}}\right\}$.

Let $d$ be valid date assignment $d$ for $I$ using only 2 slots. We assign to any literal $l$ the value "True" if and only if $(1, l)$ emits at slot 1 , and "False" otherwise. Given a clause $c_{i} \in W$, exactly one node of the form $\left(c_{i}, l\right)$ emits at slot 2 . This node is adjacent to a node $(2, l)$, which could receive the message of the request $((1, l),(2, l))$ at slot 1 only. Thus $(1, l)$ is true and $c$ is satisfied.

Reciprocally, suppose that $I_{S A T}$ admits a solution. For each literal $l$ fixed at "True", let us assign the date 1 to vertex $(1, l)$ and the date 2 to $(2, \neg l)$. Let $c_{i}$ be a clause from $W$. Date 1 is assigned to vertex $\left(1, c_{i}\right)$. Date 2 must be assigned to exactly one adjacent vertex of $\left(1, c_{i}\right)$. We can choose any couple with the corresponding literal $l$ fixed at "True". This date is available since $\left(c_{i}, l\right)$ is only adjacent to $\left(2, c_{i}\right)$ (the destination) and $(2, l)$, which has already received the message at slot 1 . Since 3 -SAT is NP-complete and 2-DAWN-request is in $\mathrm{NP}, 2$-DAWN-request is indeed a NP-complete problem.

Thus we have shown that knowledge of the routing policy plays a role in the complexity of both problems, since the limit between polynomiality and NPcompleteness is located between 2 and 3 for DAWN-path, but between 1 and 2 for DAWN-request.

\section{Solving instances with a bounded number of requests}

We give a polynomial algorithm for min-DAWN-path problem and min-DAWNrequest problem when the number of requests is bounded by above by a constant $K$. The following notation and definition will be used :

- For $i \in[1, n]$, let $\pi_{i}(t)$ denotes the $i$ th element of a n-tuple $t=\left(x_{1}, x_{2}, \ldots, x_{n}\right)$.

- The contracted form of a tuple $\left(x_{1}, x_{2}, \ldots, x_{n}\right)$ is the tuple $\left(x_{i}\right)_{(i \in[1, n]) \wedge\left(x_{i} \neq x_{i-1}\right)}$. 
We propose a polynomial algorithm to solve instances $I$ with a number of requests bounded by $K$. We build a state graph, where each vertex describes a possible state of the network at a given slot. An edge links $X$ and $Y$ if and only if one can go from state $X$ to state $Y$ or reciprocally in only one slot. For a given min-DAWN-path instance $I=\left(G, R=\left(r_{1}, r_{2}, \ldots, r_{k}\right), P\right)$ with $|R| \leq K$ the state graph $S(I)$ is defined as follows:

- the vertex set is the cartesian product $P\left(r_{1}\right) \times P\left(r_{2}\right) \cdots \times P\left(r_{K}\right)$. A vertex $X=\left(x_{1}, x_{2}, \ldots, x_{K}\right)$ indicates that for each $i \leq K$, the message of request $r_{i}$ has reached the node $\pi_{i}(X)=x_{i} \in V(G)$.

- there is an edge between $X=\left(x_{1}, x_{2}, \ldots, x_{K}\right)$ and $Y=\left(y_{1}, y_{2}, \ldots y_{K}\right)$ of $S(I)$ if and only if the simultaneous emission of nodes $\left\{x_{i} \mid x_{i} \neq y_{i}, 1 \leq i \leq K\right\}$ allows to deliver each message from $x_{i} \neq y_{i}$ to $y_{i}$ in one slot only. Formally, for $X=\left(x_{1}, x_{2}, \ldots, x_{K}\right)$ and $Y=\left(y_{1}, y_{2}, \ldots y_{k}\right),(X, Y) \in E(S(I))$ if we have, for each $i$ such that $x_{i} \neq y_{i}$ :

- $x_{i}$ and $y_{i}$ are immediately consecutive in $P\left(r_{i}\right)$,

- there is no $j \neq i$, such that $x_{j} \neq y_{j}$ and $\left\{x_{j}, y_{i}\right\} \in E(G)$,

- for each $j \neq i$ such that $x_{j} \neq y_{j}$, we have $\left|x_{i}, y_{i}, x_{j}, y_{j}\right|=4$.

The state graph $\mathrm{S}(\mathrm{I})$ of a min-DAWN-request instance $I$ is constructed according to the same method, except that the set of vertices is the set $V(G) \times$ $V(G) \times \cdots \times V(G)=V(G)^{K}$. These state graphs can be constructed in a polynomial time, since $K$ is a constant. We can distinguish two vertices in $S(I)$ : the source $\left(s_{1}, s_{2}, \ldots, s_{K}\right)$ and the sink $\left(t_{1}, t_{2}, \ldots, t_{K}\right)$ where $s_{i}$ and $t_{i}$ are respectively the source and the target of the request $r_{i}$ for any $i \in[1, K]$.

We conclude the section with this theorem:

Theorem 5. min-DAWN-path and min-DAWN-request can be solved by a polynomialtime algorithm with complexity $O\left(n^{2 K}\right)$ when the number of requests is bounded by above by a constant $K$.

Proof. (sketch of the proof) Consider $I=\left(G, R=\left(r_{1}, r_{2}, \ldots, r_{K}\right), P\right)$ a minDAWN-path instance with $|R| \leq K$, and let us construct the state graph $S(I)$.

One may check that a shortest path between the source and the sink in $S(I)$ can be associated with an optimal conflict-free date assignment and reciprocally. Such a path can be found with a $O\left(n^{2 K}\right)$ complexity algorithm.

\section{Conclusion and perspectives}

We have studied the complexity of the request satisfaction problem in a synchronous radio network. Table 1 summarises the results of this paper.

We have suggested other results [8] on particular cases i.e. on dynamic network, or when requests cannot be paused as soon as they have started. Possible perspective for this research work consist in studying the complexity of DAWNpath and DAWN-request on specific topologies, in order to discover polynomial cases even when the number of requests is unbounded. Particularly the complexity when the network is a chain is an open question (however for this case, we 


\begin{tabular}{|c|c|c|c|}
\hline \multicolumn{2}{|c|}{ DAWN-path: } & Complexity: & DAWN-request: \\
\hline \multicolumn{2}{|c|}{ Min-DAWN-path } & $\begin{array}{l}\text { NP-hard (even in trees), } \\
\text { not approximable within } \\
\text { some constant factor. }\end{array}$ & Min-DAWN-request \\
\hline \multirow[t]{2}{*}{ D-DAWN-path } & $D \leq 2$ & Polynomial & \multirow[t]{2}{*}{ D-DAWN-request } \\
\hline & $D \geq 3$ & NP-complete & \\
\hline \multicolumn{2}{|c|}{ min-DAWN-path, $|R| \leq K$} & Polynomial : $O\left(n^{2 K}\right)$ & min-DAWN-request, $|R| \leq K$ \\
\hline
\end{tabular}

Table 1.

have a constant factor approximation algorithm). Moreover, finding heuristics with performance guarantee for difficult instances constitutes a natural extension of this work.

\section{References}

1. N. Alon, A. Bar-Noy, N. Linial, and D. Peleg. A lower bound for radio broadcast. J. Comput. Syst. Sci., 43(2):290-298, 1991.

2. J.-C. Bermond, J. Galtier, R. Klasing, N. Morales, and S. Pérennes. Hardness and approximation of gathering in static radio networks. In FAWN06, Pisa, Italy, 2006.

3. J.-C. Bermond and J. Peters. Efficient gathering in radio grids with interference. In Septièmes Rencontres Francophones sur les Aspects Algorithmiques des Télécommunications (AlgoTel'05), pages 103-106, May 2005.

4. G. Chelius. Architectures et Communications dans les réseaux spontanés sans fil. PhD thesis, INSA de Lyon, INRIA Rhône Alpes, France, April 2004.

5. I. Chlamtac and S. Kutten. On broadcasting in radio networks - problem analysis and protocol design. IEEE Transactions on Communications, 33:1240-1246, 1985.

6. I. Chlamtac and O. Weinstein. The wave expansion approach to broadcasting in multihop radio network. IEEE Transaction Communication, (39):426-433, 1991.

7. T. Cormen, C. Leiserson, R. Rivest, and C. Stein. Introduction to Algorithms, 2nd edition. Dunod, 2001.

8. B. Darties. Problèmes algorithmiques et de complexité dans les réseaux sans fil. PhD thesis, LIRMM, Université Montpellier 2, France, December 2007.

9. B. Darties and J. Palaysi. Satisfaction de requêtes par affectation de dates d'émissions dans les réseaux radios. In Rencontres francophones du Parallélisme (RenPar'17), pages 157-163, 2006.

10. P. Fraigniaud and E. Lazard. Methods and problems of communication in usual networks. In Proceedings of the international workshop on Broadcasting and gossiping, pages 79-133. Elsevier North-Holland, Inc., 1994.

11. M. R. Garey and D. S. Johnson. Computers and Intractability: A guide to the theory of NP-completeness. Freeman, 1979.

12. S.M. Hedetniemi, S.T. Hedetniemi, and A.L. Liestman. A survey of gossiping and broadcasting in communication networks. Networks, 18:319-349, 1986.

13. D. R. Kowalski and A. Pelc. Centralized deterministic broadcasting in undirected multi-hop radio networks. In APPROX-RANDOM, pages 171-182, 2004. 